Impact of COVID-19 on Mohs micrographic surgery: UK-wide survey and recommendations for practice

\author{
doi: $10.1111 /$ ced.14356
}

The coronavirus disease 2019 (COVID-19) pandemic has overwhelmed and disrupted healthcare systems including dermatology services, worldwide. National directives were issued in the UK to reduce patient footfall within hospitals in anticipation of a deluge of COVID-19 admissions. ${ }^{1}$ We conducted a survey focusing on the impact of the pandemic upon Mohs micrographic surgery (MMS) practices, and invited all British Society for Dermatological Surgery (BSDS) members undertaking MMS to complete the survey over a period of 3 weeks, commencing 27 April 2020. Our findings are relevant for the restoration of MMS services and in preparation for a 'second spike' of COVID-19 cases or future pandemics.

We received 47 responses giving an estimated 52\% response rate, based on the estimate of 90 MMS surgeons in the UK (we appreciate that there may be duplication if $>1$ member from the same department answered the survey; however, practices can vary even between clinicians within a department).

In the majority of departments $(77 \%)$, doctors and nurses were redeployed to intensive care and medical wards. As a direct consequence, $49 \%$ reported that MMS services had ceased, while $36 \%$ and 15\% reported reduced and normal services, respectively. Free-text responses questioned whether the respondent felt that the level of redeployment was necessary or excessive (and if it had come at a cost to cancer services). Clinicians also highlighted that
MMS was suspended due to lack of personal protective equipment (PPE). There was confusion regarding PPE for aerosol-generating procedures and concerns that the virus may persist in fresh frozen tissue (a risk for laboratory staff). The vast majority (96\%) of respondents continuing with MMS had rationalized patients by considering highrisk tumours, comorbidities, age and patient choice.

Where post-MMS reconstruction was performed inhouse, $35 \%$ of respondents reported a decrease in the proportion of grafts/flaps (Fig. 1), 74\% reported a decrease in the proportion of external reconstructions carried out by other specialties and $81 \%$ reported increased use of dissolvable sutures, reflecting the intention of departments to reduce additional visits for suture removal. Regarding postoperative care, $71 \%$ saw no change in prescribing prophylactic antibiotics, and $29 \%$ reported an increase.

A large proportion $(40 \%)$ of respondents reported they were not reviewing patients for MMS surgery consultations or follow-up appointments, and of those who were reviewing, $79 \%$ were seeing fewer patients. Unsurprisingly, 91\% reported a decrease in face-to-face consultations, while $86 \%$ and $50 \%$ reported an increase in telephone and video consultations, respectively.

Several organizations ${ }^{2,3}$ have published guidance on treating nonmelanoma skin cancers during the pandemic but there is a paucity of guidance on MMS. The guidance pertaining to the reintroduction of elective surgical cases during the current pandemic is changing on an almost daily basis, and individual Hospital Trusts have all developed local guidelines. Thus, while position statements from national bodies such as the British Association of Dermatologists and the BSDS are desirable (70\% of survey participants felt a unified decision would be helpful), it is

Impact of COVID-19 on Mohs surgery

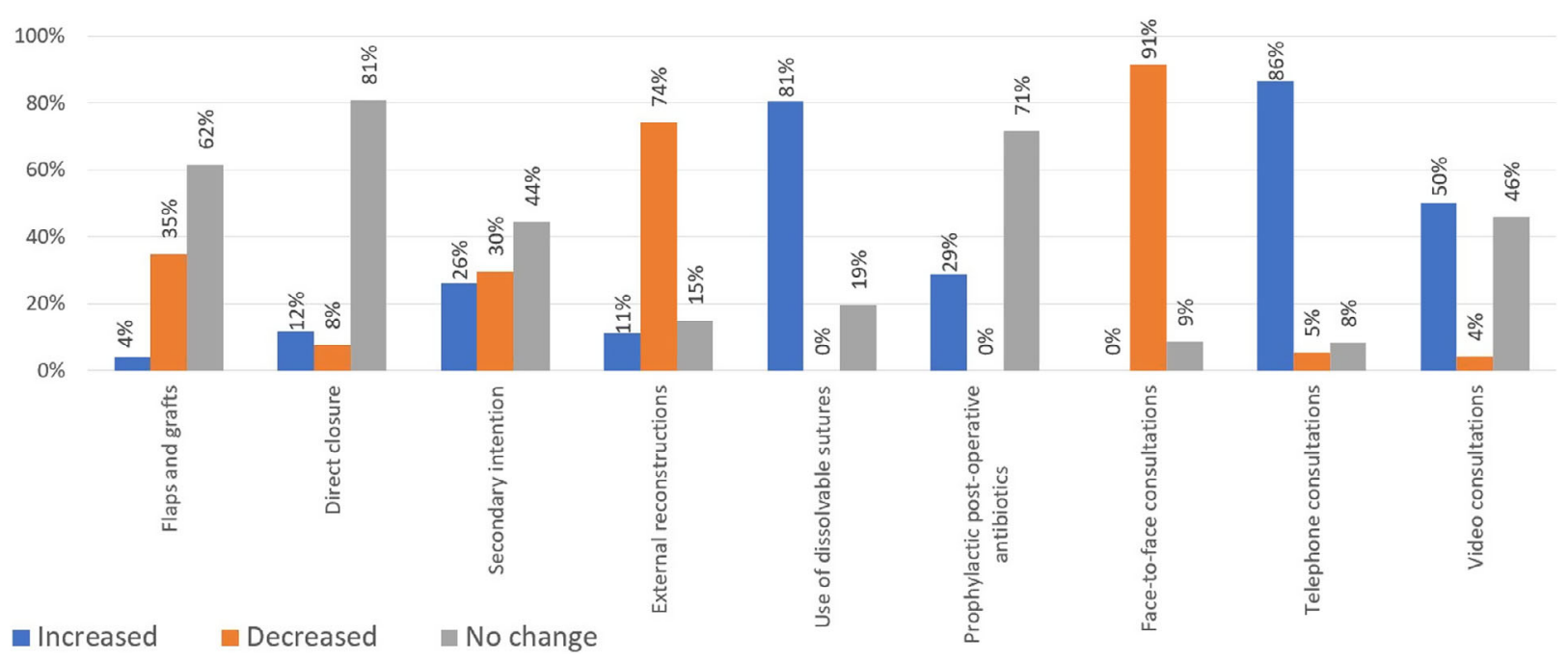

Figure 1 Survey responses on impact of COVID-19 on Mohs micrographic surgery (per proportion of respondents). 
Correspondence

Table 1 Recommendations for Mohs micrographic surgery during the COVID-19 pandemic.

\begin{tabular}{ll}
\hline Rationalize patients for & Treat patients with high-risk squamous cell carcinomas and other rarer tumours with metastatic potential \\
MMS & Treat patients for high-risk basal cell carcinomas if $<70$ years of age without significant COVID-19 risk factors \\
& Consider risk of COVID-19 to patient household members who are in a high-risk group \\
& Involve patients with informed decision-making \\
& Use multidisciplinary team approach to manage complex cases \\
Reduce infection risk & Advise patients to self-isolate for 1 week prior to MMS \\
& When patients enter department, screen them for COVID-19 symptoms with questionnaire, check their temperature \\
& and provide them with face masks \\
& Restrict accompanying visitors from entering the department \\
& Advise patients to sit at least $2 \mathrm{~m}$ apart in the waiting room \\
& Encourage patients to wash their hands and/or use sanitizers \\
& Remove potential sources of infection (e.g. books, magazines) from waiting room \\
When interacting with patients outside of theatre, use surgical mask, gloves, protective apron and visor \\
Replace face-to-face appointments with video and telephone (with photographs) consultations to reduce hospital \\
visits \\
Use dissolvable sutures to reduce inessential hospital visits \\
Use FFP3 facial mask, surgical scrub suit, surgical cap, visor, gloves and a full gown \\
Furgery \\
Falitate patients at high-risk from COVID-19 to wait in MMS theatre in between layers being taken and \\
reconstruction, rather than in the waiting room \\
Create a remote-access clinic for postoperative patients to email photographs for monitoring and/or advice
\end{tabular}

FFP, filtering face piece; MMS, Mohs micrographic surgery.

difficult for such guidance to be too prescriptive in nature, given the rapidly evolving situation. However, deferring treatment will lead to tumour progression and increased tumour burden, resulting in more challenging reconstructions and an increased likelihood of metastasis. ${ }^{4}$ We propose a number of recommendations (Table 1) to minimize risks of COVID-19 and deliver MMS services safely.

To our knowledge, this is the first nationwide survey demonstrating the impact of COVID-19 on MMS services, and it highlights significant levels of redeployment and cessation of MMS services. Although it is encouraging that departments have taken measures to reduce the number of face-to-face patient encounters and have rationalized patients for MMS, uncertainty remains about best practice. Multispeciality national guidance during this and future pandemics will help the safe and effective provision of MMS for patients.

\section{P. Nicholson ${ }^{1}$, iD F. R. Ali ${ }^{1}$ iD and R. Mallipeddi ${ }^{1}$}

${ }^{1}$ Dermatological Surgery and Laser Unit, St John's Institute of Dermatology, Guy's \& St Thomas' NHS Foundation Trust, London, $U K$

E-mail:prini13@gmail.com

Conflict of interest: the authors declare that they have no conflicts of interest.

Accepted for publication 21 June 2020

\section{References}

1 British Association of Dermatologists. COVID-19 guidance for dermatology departments, 19 March 2020. Available at: https://www.bad.org.uk/healthcare-professionals/ covid-19 (accessed 18 May 2020).
2 British Association of Dermatologists and British Society for Dermatological Surgery. COVID-19 - Skin cancer surgery guidance. clinical guidance for the management of skin cancer patients during the coronavirus pandemic. Available at: https://www.bsds.org.uk/uploads/COVID19\% 20-\%20Guidance $\% 2$ for $\% 20$ skin $\% 20$ cancer $\% 20$ surgery $\%$ 2030.03.20\%20FINAL.pdf (accessed 18 May 2020).

3 National Comprehensive Cancer Network.COVID-19 resources. Advisory statement for non-melanoma skin cancer care during the COVID-19 pandemic. Available at: https://www.nccn.org/covid-19/pdf/NCCN-NMSC.pdf (accessed 18 May 2020).

4 Der Sarkissian SA, Kim L, Veness M et al. Recommendations of dermatologic surgery during the COVID-19 pandemic. J Am Acad Dermatol 2020; 83: e29-30.

\section{Potential use of turmeric in COVID-19}

\section{doi: 10.1111/ced.14357}

Turmeric (Curcuma longa L.) is a spice that is an integral part of Asian cooking and culture, and has been used in traditional medicine systems such as Ayurveda, Unani and Siddha for centuries, owing to its wide array of medicinal properties. Curcumin, the predominant curcumoid in turmeric, influences multiple signalling pathways and been found to possess anti-inflammatory, antioxidant, antimicrobial, hypoglycaemic, wound-healing, chemopreventive, chemosensitizing and radiosensitizing properties. ${ }^{1}$ To enhance the bioavailability of curcumin, newer technologies such as adjuvant, nanoparticles, liposomes, micelles and phospholipid complexes have been evaluated 